\title{
An Unusual Presentation of Halo Nevus in a Child
}

\author{
Mikela Petkovic ${ }^{1}$, Slobodna Murat Susic ${ }^{1}$, Ruzica Jurakic Toncic ${ }^{1}$
}

1 University Department of Dermatology and Venereology, University Hospital Center and School of Medicine, Zagreb, Croatia

Key words: halo nevus, Sutton nevus, inflammatory nevus, hyperkeratotic nevus

Citation: Petkovic M, Murat Susic S, Jurakic Toncic R. An unusual presentation of halo nevus in a child. Dermatol Pract Concept. 2019;9(4):304-305. DOI: https://doi.org/10.5826/dpc.0904a13

Accepted: April 24, 2019; Published: October 31, 2019

Copyright: $@ 2019$ Petkovic et al. This is an open-access article distributed under the terms of the Creative Commons Attribution License, which permits unrestricted use, distribution, and reproduction in any medium, provided the original author and source are credited.

Funding: None.

Competing interests: The authors have no conflicts of interest to disclose.

Authorship: All authors have contributed significantly to this publication.

Corresponding author: Ruzica Jurakic Toncic, MD, Department of Dermatology and Venereology, University Hospital Center Zagreb, School of Medicine University of Zagreb, Salata 4, 10000 Zagreb, Croatia. Email: rjtoncic@gmail.com

\section{Introduction}

Nevi in children undergo morphological changes characterized by a specific dermoscopic pattern. In the majority of cases childhood nevi present dermoscopically with a globular structure, but in older children they can present with a reticular and mixed pattern. The total number of nevi increases during childhood and decreases in adulthood and leads to almost complete disappearance of nevi in advanced age (with the exception of dermal and congenital nevi). Nevi involute in 3 different pathways: via "halo" phenomenon, a regression pattern, and a gradual involution (no evidence of regression pattern or halo phenomenon). Two different perilesional phenomena of melanocytic nevus can be observed: depigmentation, found in halo nevus (HN), and eczema, related to Meyerson nevus. Halo phenomenon is a result of an immunological mechanism in which nevocellular antigens are recognized by the immune system, both humoral and cell-mediated. HN, also known as Sutton nevus, is a benign type of nevus commonly found in children that presents with a central pigmented part surrounded by a hypopigmented zone.

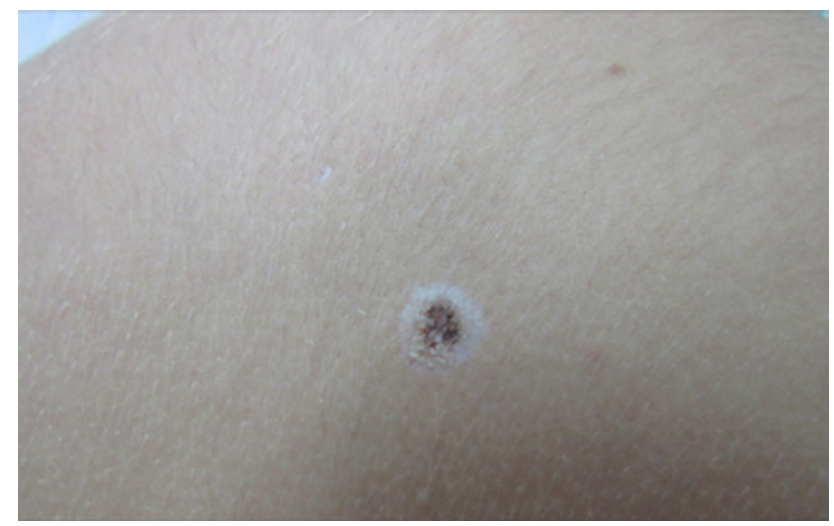

Figure 1. The patient presented with sharply demarcated peripheral hypopigmentation with central hyperkeratotic nevus. [Copyright: (C)2019 Petkovic et al.]

\section{Case Presentation}

We report an unusual presentation of $\mathrm{HN}$ in 11-year-old girl. A clinically heavily pigmented verrucous lesion localized on the back was found, measuring $5 \times 5 \mathrm{~mm}$, with $3 \mathrm{~mm}$ of surrounding hypopigmentation. Clinically the lesion was verrucous, which is not common in $\mathrm{HN}$ (Figure 1). Dermoscopy showed criteria for a melanocytic lesion, central nonspecific 


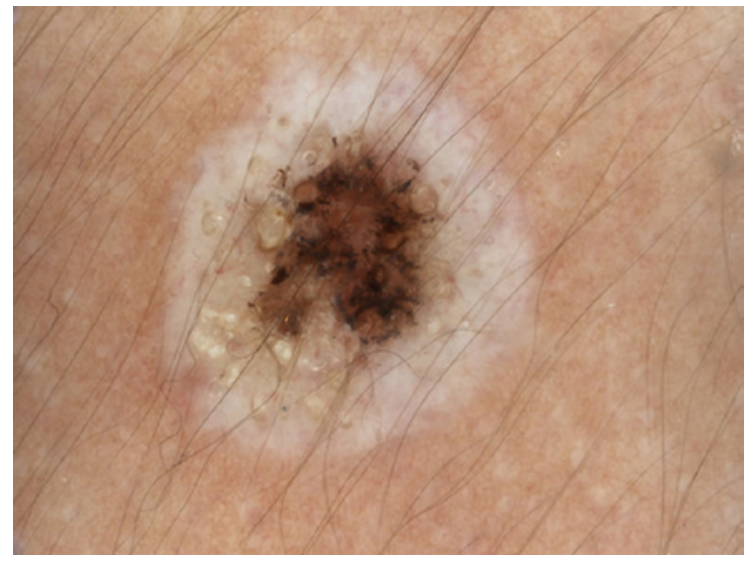

Figure 2. Dermoscopy showed centrally nonspecific dermoscopic structure and peppering surrounded by intensive white color and keratin plugs. [Copyright: @2019 Petkovic et al.]

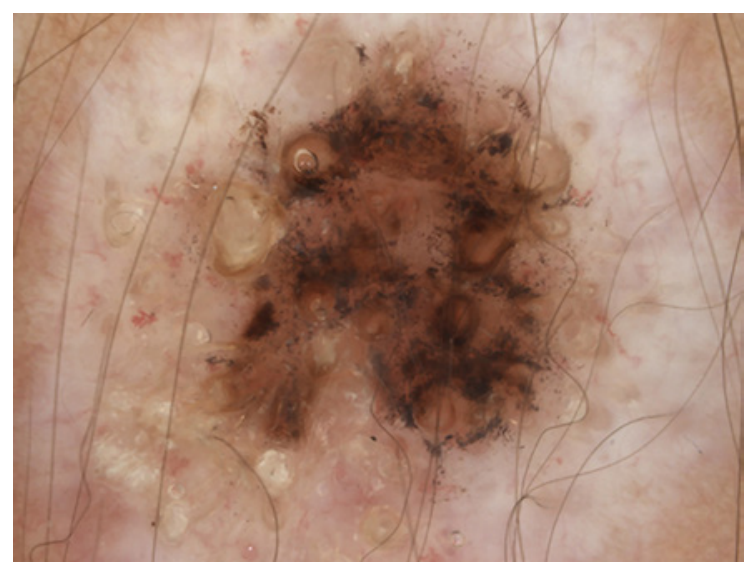

Figure 3. On higher magnification atypical vessels were found, surrounded by a peripheral rim of intense hypopigmentation. [Copyright: @2019 Petkovic et al.]

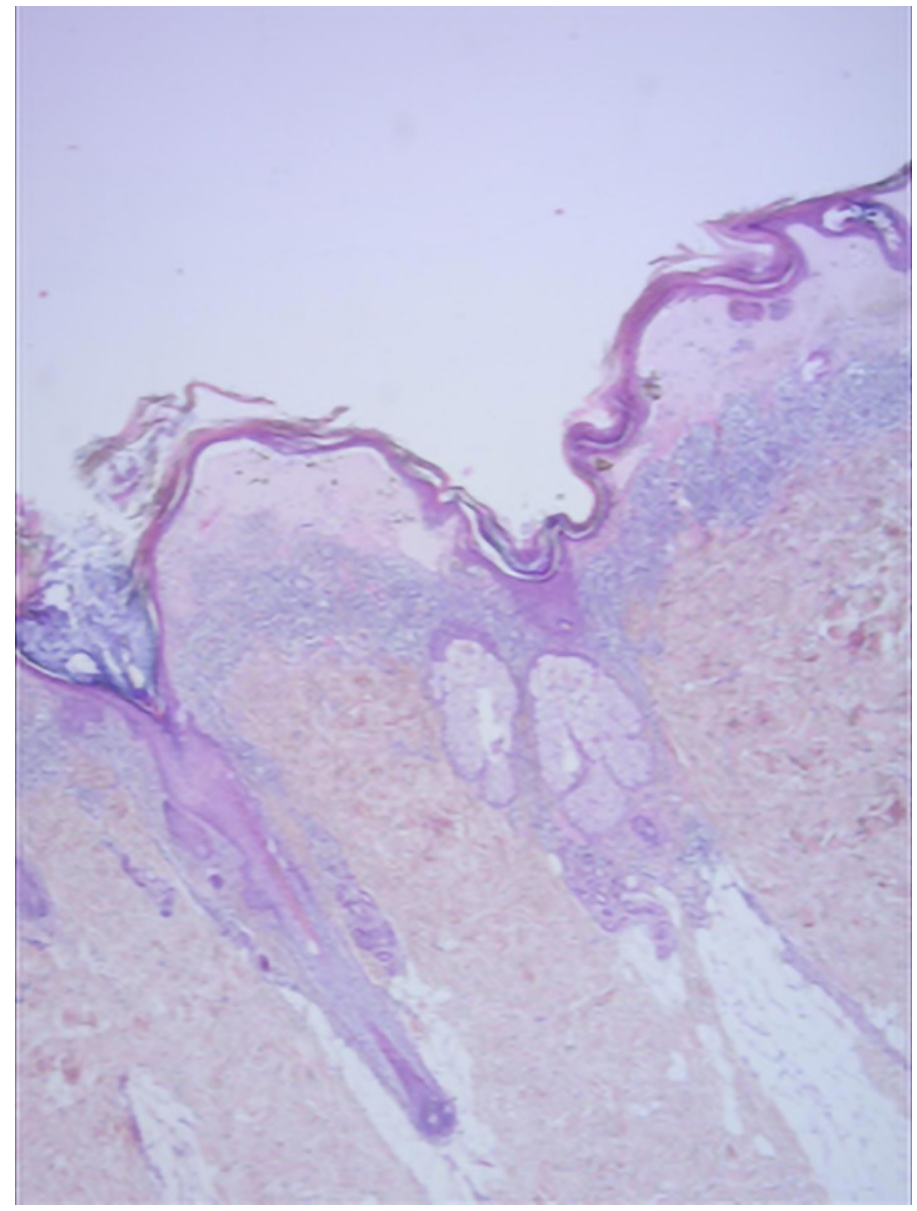

Figure 4. On magnification $(\times 15)$, histopathology showed focally thinned, hyperkeratotic epidermis with small nests of nevus cells with abundant melanin in the basal layer of the epidermis. In the papillary dermis significant fibrosis and edema were found, along with evenly distributed lymphocytic infiltrate and clusters of pigmentophages. [Copyright: @2019 Petkovic et al.] dermoscopic structure with peppering, suggesting regression of lesion. Peripherally intensive white color and keratin plugs were found (more pronounced under nonpolarized dermoscopy) (Figures 2 and 3). Duration of the lesion was unknown. The clinical and dermoscopic presentation were primarily indicative of a benign lesion, but it was excised because of its atypical clinic appearance. Histology showed focally thinned, hyperkeratotic epidermis. Small nests of nevus cells with abundant melanin were found in the basal layer of the epidermis. In the papillary dermis, significant fibrosis and edema were found, along with evenly distributed lymphocytic infiltrate and clusters of pigmentophages (Figure 4). After clinicohistopathological correlation, the diagnosis of "inflammatory and hyperkeratotic halo nevus of children" was established.

\section{Conclusions}

This is a case of an unusual presentation of HN with hyperkeratotic changes of the epidermis. This observation was described by 1 group of authors [1]. The existence of this entity is still elusive, since there are no other described cases in children or adults to our knowledge. Hyperkeratosis, an epidermal hyperplasia, is not a common finding in nevus, but it can be observed and hyperkeratotic changes found in nevus are thought to be a result of higher expression of Ki 67 [2].

The first description and series of case reports of hyperkeratotic $\mathrm{HN}$ in children was done by Patrizi et al [1]. Halo phenomenon can be observed in some other benign or malignant entities; therefore, all lesions with depigmentation should be examined with caution. It remains elusive why only few halo nevi develop hyperkeratotic changes, which is observed in our case. Awareness of its existence is important in order to avoid unnecessary surgery in children.

\section{References}

1. Patrizi A, Neri I, Sabattini E, Rizzoli L, Misciali C. Unusual inflammatory and hyperkeratotic halo naevus in children. $\mathrm{Br} \mathrm{J}$ Dermatol. 2005;152(2):357-360.

2. Horenstein MG, Prieto VG, Burchette JL, Shea CR. Keratotic melanocytic nevus: a clinicopathologic and immunohistochemical study. J Cutan Pathol. 2000;27(7):344-350. 